\title{
Monitoring of adipogenic differentiation at the single-cell level using atomic force microscopic analysis
}

\author{
Young-Nam Kwon ${ }^{\mathrm{a}, *}$, Won Kon Kim ${ }^{\mathrm{b}, *}$, Sang-Hak Lee ${ }^{\mathrm{c}}$, Keewon Kim ${ }^{\mathrm{a}}$, Eun Young Kim ${ }^{\mathrm{b}}$, \\ Tai Hwan $\mathrm{Ha}^{\mathrm{d}}$, HyoukSoo Han ${ }^{\mathrm{a}}$ and Kwang-Hee Bae ${ }^{\mathrm{b}, * *}$ \\ a Samsung Advanced Institute of Technology, Kyung-gi, Korea \\ ${ }^{\mathrm{b}}$ Analytical Science Group, Medical Proteomics Research Center, KRIBB, Daejeon, Korea \\ ${ }^{\mathrm{c}}$ Department of Chemistry, Seoul National University, Seoul, Korea \\ ${ }^{\mathrm{d}}$ Immunotherapy Research Center, KRIBB, Daejeon, Korea
}

\begin{abstract}
Adipogenesis plays an important role in energy homeostasis by storing excess energy as lipid droplets. However, these reservoirs are implicated in a host of major human health problems, such as obesity. Elucidation of the mechanisms underlying adipogenesis is thus crucial to overcome these problems. The preadipocyte cell lines represent an optimal model to examine adipogenesis. Cells differentiate into adipocytes with various speeds of conversion and fat accumulation. Here, we have presented a novel method for detecting adipogenic differentiation at the single-cell level using atomic force microscopic analysis. Data obtained with this method revealed a good correlation between membrane stiffness and the degree of adipogenic differentiation. Although we could not determine the underlying cause for membrane stiffness reduction during adipogenic differentiation, the technique clearly offers advantages over the existing detection systems, such as lipid drop staining and extraction. Furthermore, the degree of adipogenic differentiation at the single-cell level can be detected with this method.
\end{abstract}

Keywords: Adipocyte, adipogenesis, AFM, differentiation, stiffness

\section{Introduction}

Adipocytes are highly specialized cells that play an important role in energy homeostasis by harboring energy reservoirs as lipid droplets [2,7,11]. However, these reservoirs are involved in a variety of human health problems, since excessive or insufficient energy reserves result in metabolic disorders, such as obesity and lipodystrophy [5,16]. Obesity is a grave medical issue in developed countries due to its association with several dangerous diseases, including type II diabetes, hypertension, coronary heart disease, stroke and cancer. Adipogenesis involves the formation of preadipocytes from mesenchymal progenitor cells and their subsequent differentiation into adipocytes $[6,8,19]$.

3T3-L1, a preadipocyte cell line derived from $3 \mathrm{~T} 3$ cells, is used as an in vitro model of adipocytes. 3T3-L1 cells display fibroblast-like morphology and the cells differentiate into adipocytes under the

\footnotetext{
${ }^{*}$ Both authors contributed equally to this study.

** Corresponding author: Kwang-Hee Bae, PhD, Medical Proteomics Research Center, KRIBB, 50 Eon-dong, Yusung-gu, Daejeon 305-806, Korea. Fax: +82 42860 4269; E-mail: khbae@kribb.re.kr.
} 
appropriate conditions. However, significant cell-to-cell variations in cell morphology and lipid drop accumulation have been observed for differentiating preadipocytes. Therefore, efficient monitoring of the cells during adipogenesis is necessary to elucidate the novel molecular states and responses to external stimuli or perturbations.

Atomic force microscopy (AFM) is an extremely high-resolution method for determining the topography and mechanical properties of materials, including cells. This method provides a powerful tool for elucidating morphological aspects of cell structures at nanometer scale resolution and their rheological properties in living cells $[3,12,14,18]$. Mechanical elasticity of materials, usually measured as stiffness, is one of the most critical parameters, and can be assessed with AFM. Recent reports have suggested that AFM can be used to determine the elasticity of living cells with nanoscale resolution [1,4,9,20,21]. Moreover, tumor cells have different elastic compliance, compared to normal cells [15].

Major changes occur in overall appearance and plasma membrane organization upon differentiation of preadipocyte cells into mature adipocytes. In this study, we examined membrane stiffness during differentiation of the 3T3-L1 preadipocyte cell line into mature adipocytes using AFM. Our data indicate that membrane stiffness and differentiation are well correlated. This method provides a novel tool to detect adipogenic differentiation at the single-cell level, and may be used in combination with the existing methods for studying adipogenic differentiation as a precise means to develop agents for treating obesity.

\section{Materials and methods}

\subsection{T3-L1 cell culture and adipogenesis}

The preadipocyte cell line, 3T3-L1, derived from mouse embryo fibroblasts, was cultured in growth medium (high glucose Dulbecco's modified Eagle's medium (DMEM) containing 1\% antibioticantimycotic solution and $10 \%$ bovine calf serum (BCS), Gibco-Invitrogen) at $37^{\circ} \mathrm{C}$ in a humidified atmosphere with $5 \% \mathrm{CO}_{2}$.

3T3-L1 cells were induced to differentiate into mature adipocytes using a previously described protocol $[8,10,11]$. Briefly, at 2 days after confluence (day 0 ), cells were initially incubated in differentiation medium composed of DMEM, $10 \%$ FBS and MDI, a differentiation cocktail of $0.5 \mathrm{mM}$ isobutylmethylxanthine (IBMX), $1 \mu \mathrm{M}$ dexamethasone and $10 \mu \mathrm{g} / \mathrm{ml}$ insulin (Sigma), and switched to maintenance medium composed of DMEM, 10\% FBS and $10 \mu \mathrm{g} / \mathrm{ml}$ insulin. The medium was replenished every other day.

\subsection{Oil-Red-O staining}

Lipid droplets in differentiating or mature adipocytes were stained using the Oil-Red-O method, as described previously $[8,10]$. In brief, cultured cells were washed twice with PBS, fixed for 30 min with $10 \%$ formalin and washed twice with distilled water prior to staining. Lipid droplets within the cell were stained for 30 min using $0.3 \%$ filtered Oil-Red-O solution in 60\% isopropanol (Sigma). Cells were washed twice with distilled water, and micrographs obtained. Oil-Red-O staining was quantified, as described in an earlier report [17]. After elution from fixed cells with absolute isopropanol, extracted dye was measured with a GeneQuant 1300 spectrophotometer (GE Healthcare) at $510 \mathrm{~nm}$. 


\subsection{Flow cytometry}

Cells were washed 5 times with $1 \mathrm{ml}$ washing buffer, and cell pellets dissolved in DPBS containing $10 \%$ FBS. Next, the cells filtered with $100 \mu$ m Cell Strainer (BD Falcon). Fluorescence was determined with FACS Aria (BD Bioscience) for 10,000 events.

\subsection{Atomic force microscopy}

A NanoWizard II (JPK instruments) AFM mounted on an inverted optical microscope was used for all experiments. The AFM had a maximum horizontal scanning range of $100 \times 100 \mu \mathrm{m}^{2}$ and a vertical range of $15 \mu \mathrm{m}$. Silicon nitride cantilevers (AdvancedTECTM FM AFM tips, Nanosensors) with a nominal spring constant of $2.8 \mathrm{~N} / \mathrm{m}$ were used. At the specified range, the value of the spring constant is $0.7-9.0 \mathrm{~N} / \mathrm{m}$. All AFM measurements were obtained in contact mode in liquid. The absolute value of the slope $(\mathrm{N} / \mathrm{m})$ in this curve indicates the different levels of force applied over the same distance (approaching the cell) and thus provides a qualitative measure of stiffness and elasticity.

\subsection{Statistical analysis}

Quantitative data were processed with Origin version 8. The means and standard deviations are illustrated in the figures. All quantitative data were analyzed using an independent Student's $t$-test, and considered significant at $P<0.05$.

\section{Results and discussion}

\subsection{Adipogenic differentiation of 3T3-L1 cells and Oil-Red-O staining}

Microscopic examination of cellular lipid droplet formation and lipid staining by Oil-Red $\mathrm{O}$ has been widely used to assess fat cell development and maturation during adipogenesis. We performed the microscopic analysis and lipid staining with Oil-Red $\mathrm{O}$ to monitor the degree of lipid accumulation during adipogenesis. As shown in Fig. 1, intracellular lipid accumulation is continuously increased during adipogenesis of 3T3-L1 cells. Contrast microscopy used to monitor morphological changes is not able to discriminate the accumulation of lipid droplets at the single-cell level. Furthermore, quantitative analysis of the degree of adipogenic differentiation is problematic. It is also difficult to monitor adipogenic differentiation at the single-cell level. On the other hand, Oil-Red-O staining provides a quantitative tool for assessing the amount of total lipid droplets.

\subsection{FACS characterization of adipogenic differentiation of 3T3-L1}

Lee and co-workers reported a simple, sensitive and quantitative novel method to assess lipid accumulation in fat cells using flow cytometry [13]. This procedure allows monitoring of the degree of intracellular lipid accumulation in a precise and rapid manner. The method was additionally used to examine adipogenic differentiation of 3T3-L1 cells. Under our experimental conditions, we observed a good correlation between the degree of adipogenic differentiation and SSC-H (side scatter) value of flow cytometry (Fig. 2). Notably, cells are heterogeneous in their response to adipogenic agents with regard to speed of differentiation and degree of lipid accumulation. Although the reason for the correlation between adipogenic differentiation and SSC-H value is unclear at present, this method appears effective for monitoring adipogenic differentiation at the single-cell level. 


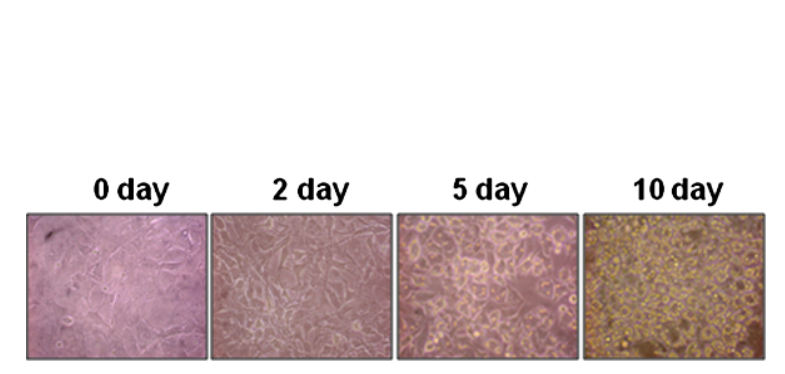

(a)

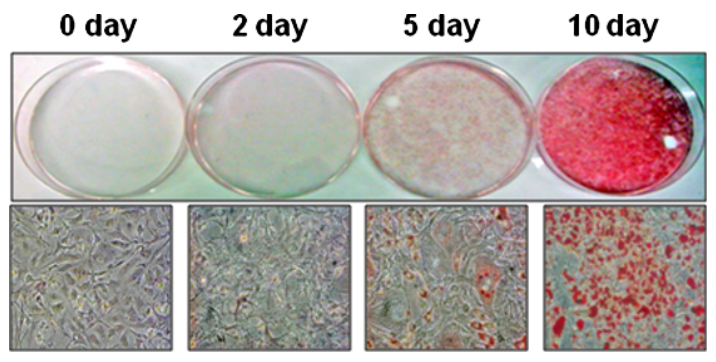

(b)

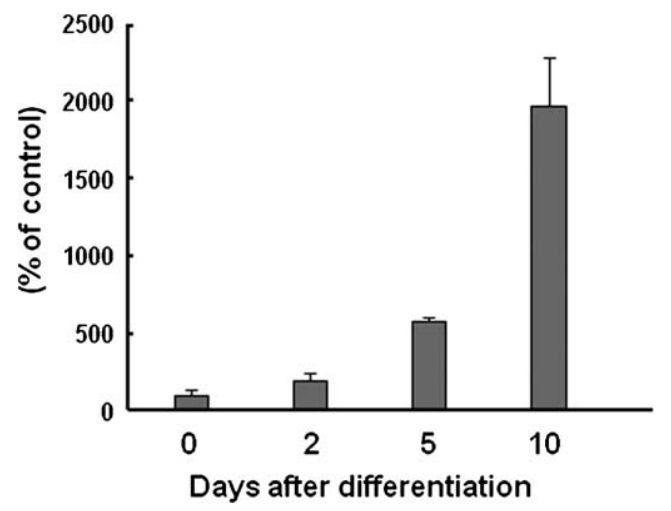

(c)

Fig. 1. Monitoring of adipogenic differentiation. (A) Adipogenic differentiation was monitored using bright-field microscopy. As shown, lipid droplets were detected at the late stages of adipogenesis. (B) Lipid droplets were stained using the Oil-Red-O method, as described previously (Kim et al., 2009). (C) Quantification with Oil-Red-O staining. After elution from fixed cells with absolute isopropanol, extracted dye was measured with a GeneQuant 1300 spectrophotometer (GE Healthcare) at $510 \mathrm{~nm}$. (Colors are visible in the online version of the article; http://dx.doi.org/10.3233/SPE-2012-0566.)

\subsection{Monitoring adipogenic differentiation by assessing membrane stiffness using AFM}

In terms of major changes in the overall appearance and plasma membrane organization during adipogenesis, the stiffness of cells undergoing adipogenic differentiation (days 0,2,5 and 10) was investigated using AFM tips with the same spring constant. The technique of approaching the AMF probe to 3T3-L1 cells was used to measure the hardness of cells during adipogenic differentiation. Live cells were approached in a liquid environment using AFM equipment with a triangular silicon probe. Force-distance curves (FDC) of cells were acquired at various stages of adipogenic differentiation. AFM analysis disclosed that 3T3-L1 cells before adipogenic differentiation (day 0) exhibited a flat slope of FDC. On the other hand, 3T3-L1 cells at late stage of differentiation (day 10) showed a sharper FDC slope and significantly increased softness, compared with 3T3-L1 cells, at day 0 (Fig. 3A). In addition, the force curves of 30-60 surfaces of individual cells at various stages of adipogenic differentiation were obtained and the mean value of the slope of the FDC plotted (Fig. 3B). The results clearly demonstrated a significant correlation between adipogenic differentiation and cell stiffness/shrinking patterns. No significant differences were evident in the levels of mechanical stiffness between cells at days 0 and 2. However, 3T3-L1 cells obtained from late stages of adipogenesis (days 5 and 10) showed a significantly dispersed pattern in the mean value of the FDC slope, indicating significant cell-to-cell variations in morphology and lipid drop accumulation. 
0 day

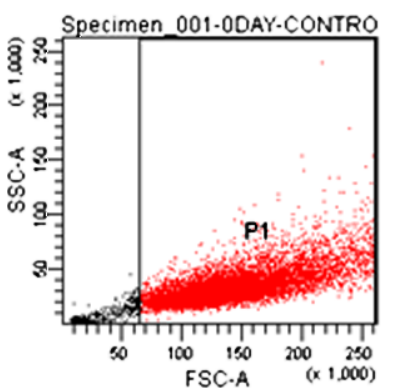

12 day

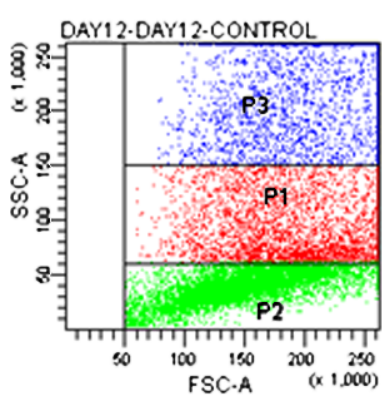

2 day

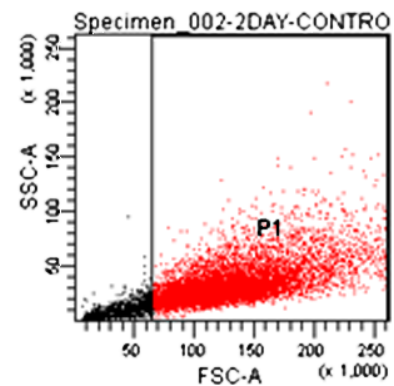

(a)
12 day

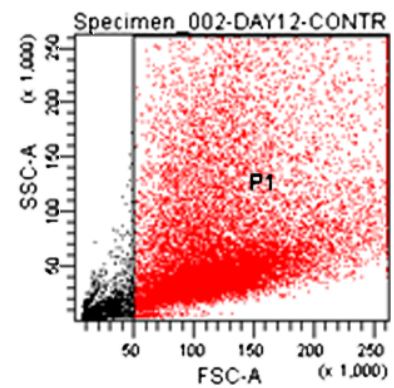

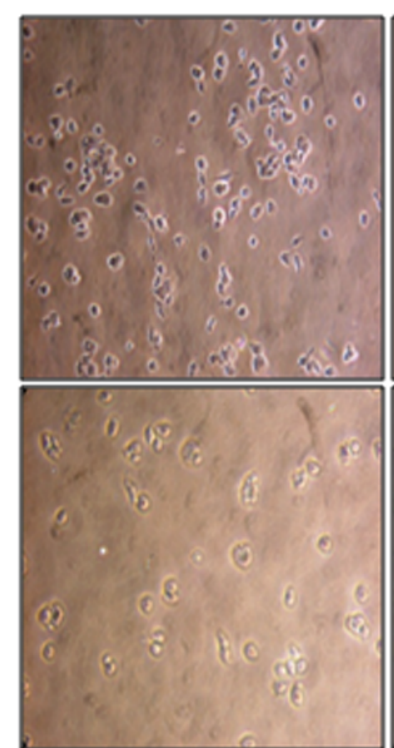

P3

(b)

Fig. 2. Increased granularity (SSC-H) of 3T3-L1 during adipogenesis. (A) Dot plots of side scatter (SSC; $x$-axis) versus forward scatter (FSC; $y$-axis) of 3T3-L1 cells generated from flow cytometric analysis at different time-points of adipogenesis. (B) Light microscopic examination of 3T3-L1 cells with different SSC-H values. (Colors are visible in the online version of the article; http://dx.doi.org/10.3233/SPE-2012-0566.)

\section{Conclusions}

Our findings indicate that 3T3-L1 preadipocyte cells possess characteristic mechanical biomarkers that are detectable with AFM analysis. Although the detailed mechanisms underlying the association between membrane stiffness and adipogenic differentiation remain to be established, the method presented in this study may be used as an effective monitoring tool for assessing the degree of adipogenic differentiation at the single-cell level. 


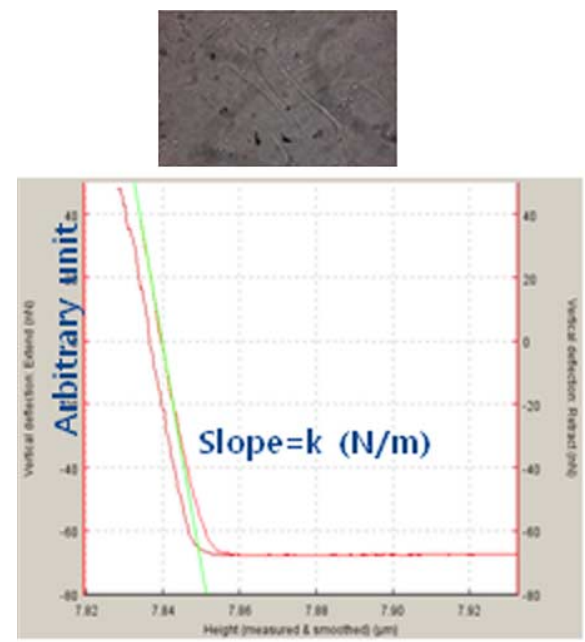

Day 0
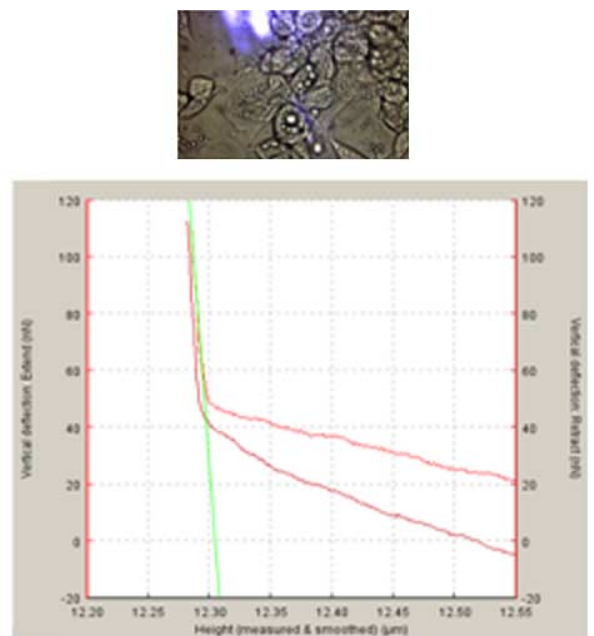

Day 10

(a)

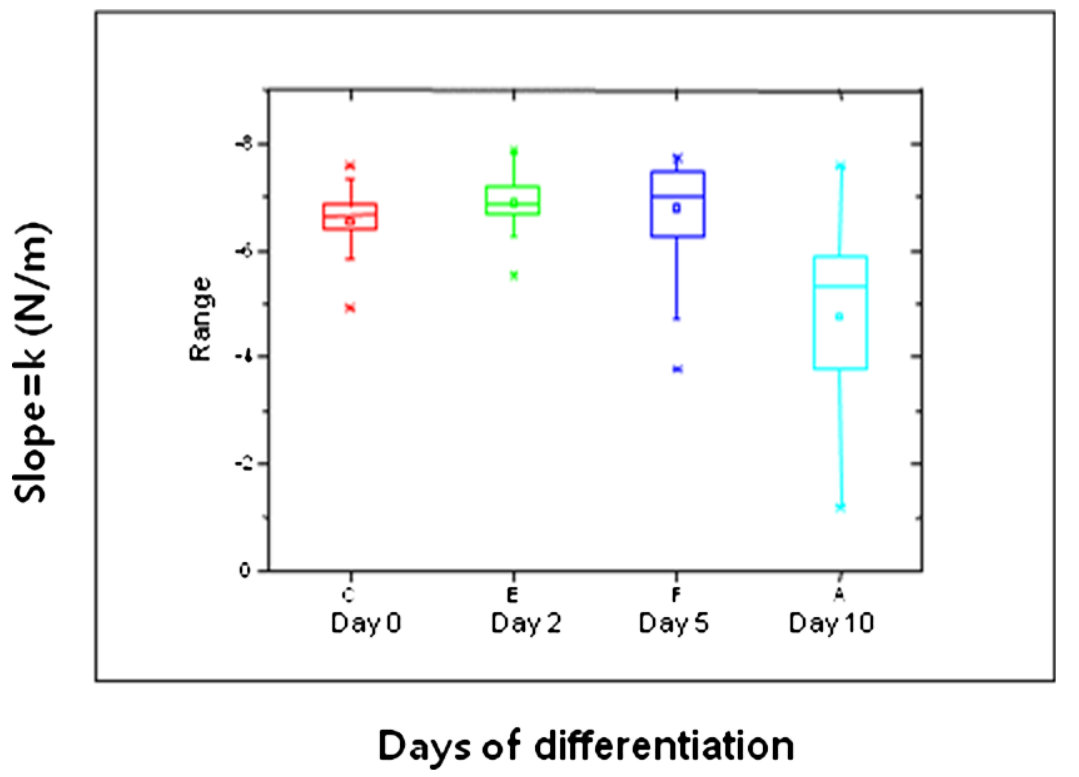

(b)

Fig. 3. Evaluation of mechanical properties during adipogenic differentiation by assessing membrane stiffness using AFM. (A) 3T3-L1 cells at the late stage of differentiation (day 10) showed a sharper FDC slope and significantly increased softness, compared with 3T3-L1 cells at day 0 (control). (B) The mean value of the elastic moduli (mechanical property) of day 10 cells was significantly lower than that of 3T3-L1 cells at the early stages of adipogenic differentiation (days 0 and 2) and showed a significantly dispersed pattern in the mean value of the FDC slope. (Colors are visible in the online version of the article; http://dx.doi.org/10.3233/SPE-2012-0566.)

\section{Acknowledgements}

We are grateful to Drs. Do Hee Lee, Sayeon Cho, Seung-Wook Chi, Sung Goo Park and Byoung Chul Park for careful reading of the manuscript. We also thank Dr. Sang J. Chung for technical assistance and helpful discussions. This work was supported by National Agenda Program of KRCF, research 
program (grant nos. 2011-0027634, 2011-0004447 and 2011-0020507) of Korea Research Foundation and KRIBB.

\section{References}

[1] S. Choi, Y. Cheong, H.J. Lee, S.J. Lee, K.H. Jin and H.K. Park, J. Nanosci. Nanotechnol. 11 (2011), 6382-6388.

[2] P. Cornelius, O.A MacDougald and M.D. Lane, Annu. Rev. Nutr. 14 (1994), 99-129.

[3] D. Docheva, D. Padula, C. Popov, W. Mutschler, H. Clausen-Schaumann and M. Schieker, J. Cell. Mol. Med. 12 (2008), 537-552.

[4] J.Y. Fan, J.-L. Carpentier, E.V. Obberghen, C. Grunfeld, P. Gorden and L. Orci, J. Cell Sci. 61 (1983), 219-230.

[5] A. Garg, J. Clin. Endocrinol. Metab. 85 (2000), 1776-1782.

[6] F.M. Gregoire, C.M. Smas and H.S. Sul, Physiol. Rev. 78 (1998), 783-809.

[7] C.S Hwang, T.M. Loftus, S. Mandrup and M.D. Lane, Annu. Rev. Cell Dev. Biol. 13 (1997), 231-259.

[8] H. Jung, W.K. Kim, D.H. Kim, Y.S. Cho, S.J. Kim, S.G. Park, B.C. Park, H.M. Lim, K.-H. Bae and S.C. Lee, Biochem. Biophys. Res. Comm. 383 (2009), 252-257.

[9] C.-L. Kao, L.-K. Tai, S.-H. Chiou, Y.-J. Chen, K.-H. Lee, S.-J. Chou, Y.-L. Chang, C.M. Chang, S.-J. Chen, H.-H. Ku and H.-Y. Li, Stem Cell. Dev. 19 (2010), 247-257.

[10] W.K. Kim, H. Jung, E.Y. Kim, D.H. Kim, Y.S. Cho, B.C. Park, S.G. Park, Y. Ko, K.-H. Bae and S.C. Lee, Mol. Biol. Cell 22 (2011), 4883-4891.

[11] W.K. Kim, H. Jung, D.H. Kim, E.Y. Kim, J.-W. Chung, Y.-S. Cho, S.G. Park, B.C. Park, K.-H. Bae and S.C. Lee, J. Cell Sci. 122 (2009), 4160-4167.

[12] A. Labernadie, C. Thibault, C. Vieu, I. Maridonneau-Parini and G.M. Charriere, Proc. Natl. Acad. Sci. USA 107 (2008), 21016-21021.

[13] Y.-H. Lee, S.-Y. Chen, R.J. Wiesner and Y.-F. Huang, J. Lipid Res. 45 (2004), 1162-1167.

[14] V. Lulevich, H.Y. Yang, R.R. Isseroff and G.Y. Liu, Ultramicroscopy 110 (2010), 1435-1442.

[15] V. Lulevich, T. Zink, H.-Y. Chen, F.-T. Liu and G.Y. Liu, Langmuir 22 (2006), 8151-8155.

[16] T.C. Otto and M.D. Lane, Crit. Rev. Biochem. Mol. Biol. 40 (2005), 229-242.

[17] I. Ramirez, Appetite 18 (1992), 193-206.

[18] C. Roduit, F.G. Goot, P.D.L. Rios, A. Yersin, P. Steiner, G. Dietler, S. Catsicas, F. Lafont and S. Kasas, Biophys. J. 94 (2008), 1521-1532.

[19] E.D. Rosen and B.M. Spiegelman, Annu. Rev. Cell Dev. Biol. 16 (2000), 145-171.

[20] J. Storch, S.L. Shulman and A.M. Kleinfeld, J. Biol. Chem. 264 (1989), 10527-10533.

[21] T. Yan, R. Sun, H. Deng, B. Tan and N. Ao, Scanning 31 (2009), 246-252. 


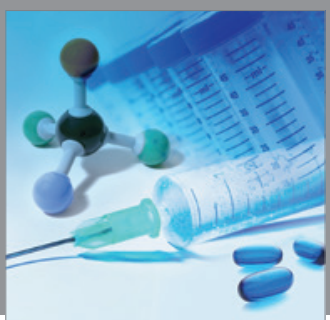

International Journal of

Medicinal Chemistry

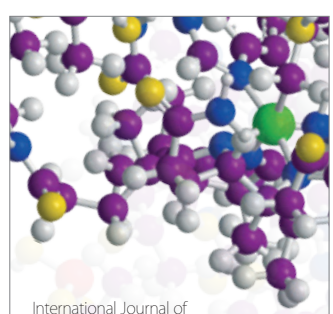

Carbohydrate Chemistry

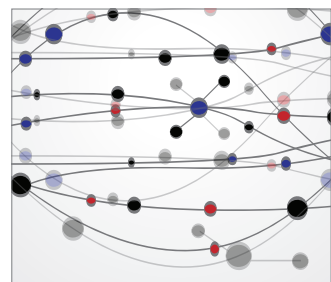

The Scientific World Journal
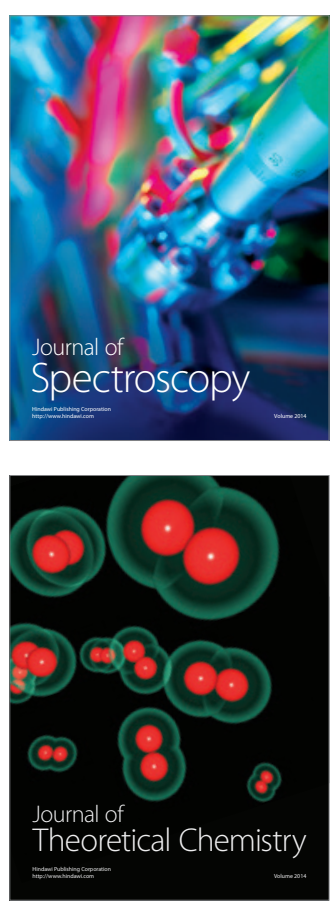
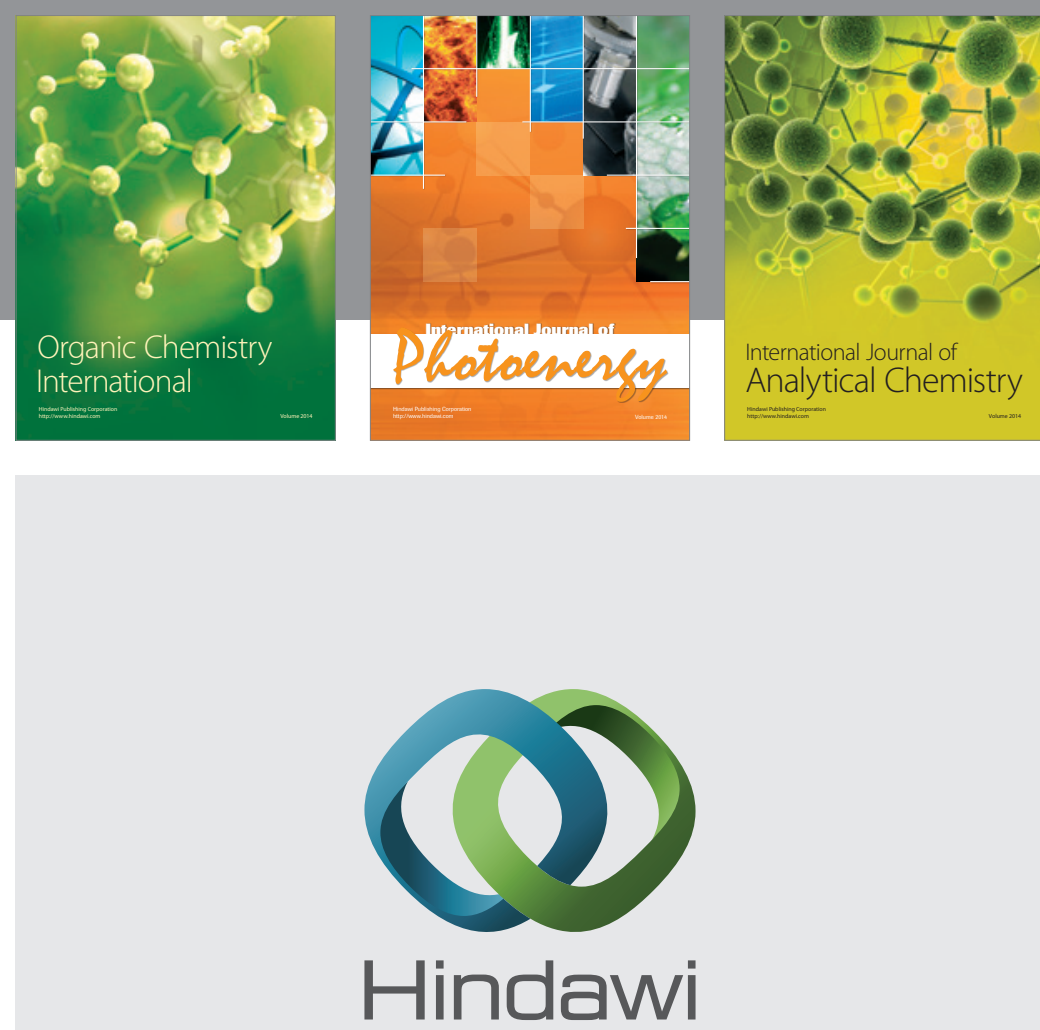

Submit your manuscripts at

http://www.hindawi.com
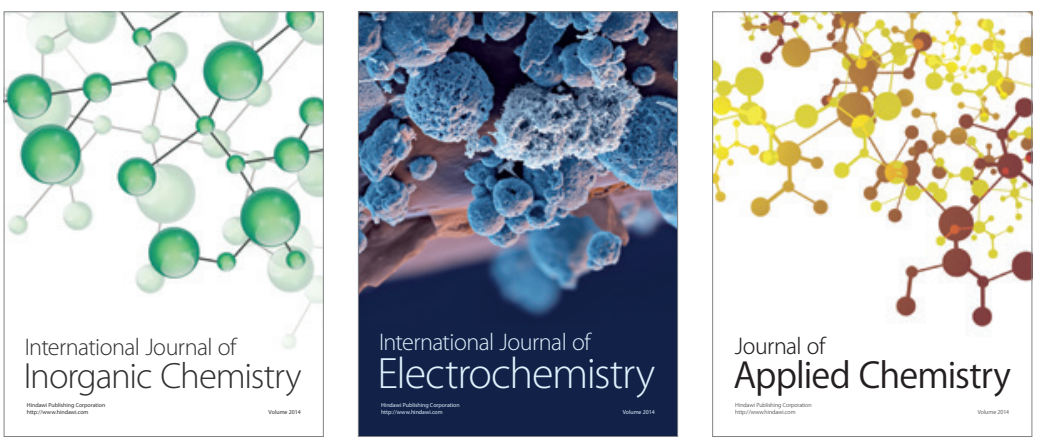

Journal of

Applied Chemistry
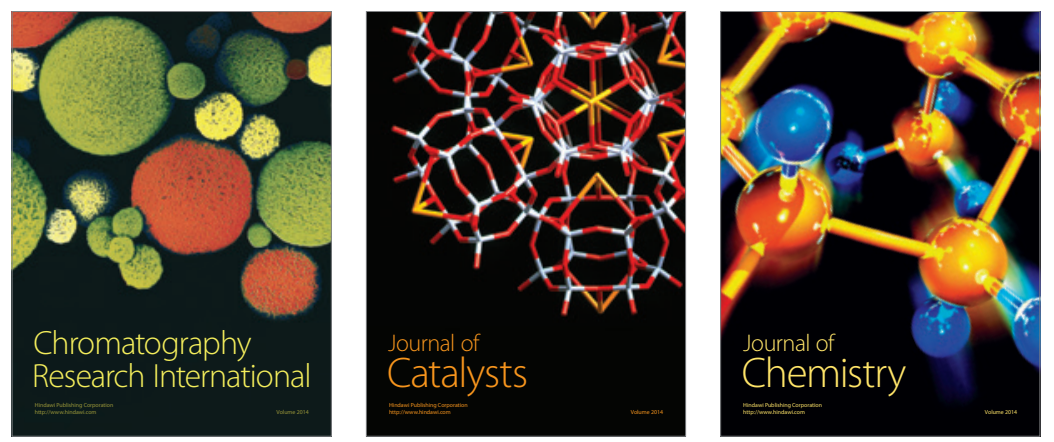
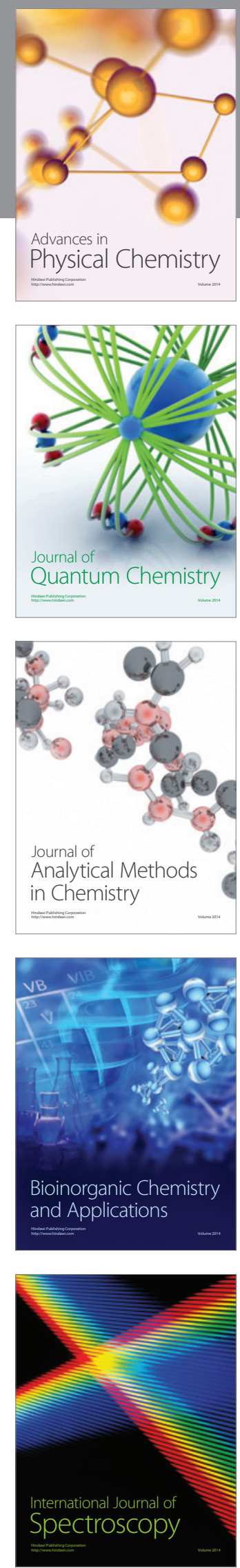